\title{
ON THE GLOBAL DIMENSION OF A RING MODULO ITS NILPOTENT RADICAL
}

\author{
ELLEN KIRKMAN AND JAMES KUZMANOVICH
}

(Communicated by Donald Passman)

\begin{abstract}
For any preassigned integer $n \geq 3$, a Noetherian affine PI ring $R$ is constructed with $\mathrm{gl} \operatorname{dim} R \leq 3$, but $\operatorname{gl} \operatorname{dim}(R / N(R))=n$. A second similar ring is constructed with $\operatorname{gl} \operatorname{dim} R \leq 5$ and $\operatorname{gl} \operatorname{dim}(R / N(R))=\infty$.
\end{abstract}

The question of whether $\operatorname{rgl} \operatorname{dim}(R / N(R)) \leq \operatorname{rgl} \operatorname{dim} R$ in a right Noetherian ring $R$, where $N(R)$ is the nilpotent radical of $R$, is an old one; see [ReSS, p. 294]. Eilenberg, Nagao, and Nakayama [ENN] provided examples to show that $\mathrm{rgl} \operatorname{dim}(R / I)$ can exceed $\operatorname{rgl} \operatorname{dim} R$ by an arbitrarily large amount for a nilpotent ideal $I(I \varsubsetneqq N(R))$ even in an Artinian hereditary ring; yet for Noetherian right hereditary rings, Small $[\mathbf{S}$, Theorem 4] showed that $R / N(R)$ is hereditary, and results of Gordon and Small [GS] give the same result for more general hereditary rings (e.g., for a right hereditary ring with no infinite set of orthogonal idempotents). In these last two cases, the hereditary $\operatorname{ring} R$ is isomorphic to a triangular matrix ring. Fields [F2] showed that for any trianglar matrix ring $R=\left[\begin{array}{cc}R_{1} & M \\ 0 & R_{2}\end{array}\right]$, $\operatorname{rgl} \operatorname{dim} R_{i} \leq \operatorname{rgl} \operatorname{dim} R$, so that a triangular matrix ring will not provide a counterexample to $\operatorname{rgl} \operatorname{dim}(R / N(R)) \leq \operatorname{rgl} \operatorname{dim} R$. In this paper we construct examples which show that for even a quite nice Noetherian ring $R$ of finite global dimension, $\mathrm{gl} \operatorname{dim}(R / N(R))$ can be arbitrarily larger than $\mathrm{gl} \operatorname{dim} R$, or even infinite.

We begin by recalling the Eilenberg, Nagao, and Nakayama examples, which will be used in our construction.

EXAMPLE 1 [ENN]. Let $T=T_{m}(k)$ denote the ring of $m \times m$ upper triangular matrices over a field $k$, and $N(T)$ the ideal of strictly upper triangular matrices; then $T$ is a hereditary ring and for $I=(N(T))^{2}, \operatorname{gl} \operatorname{dim}(T / I)=m-1$.

EXAMPLE 2 [ENN]. Let

$$
T=\left[\begin{array}{ccc}
a & b & c \\
0 & d & e \\
0 & 0 & a
\end{array}\right] \leq T_{3}(k)
$$

and $I=(N(T))^{2}$. Then $\mathrm{gl} \operatorname{dim} T=2$ but $\mathrm{gl} \operatorname{dim}(T / I)=\infty$.

We will produce rings $R$ of finite global dimension by choosing them so that they are left subidealizers in a ring $S$ of finite global dimension at an ideal $A$ of $R$ with $\operatorname{gl} \operatorname{dim}(R / A)<\infty$. In saying that $R$ is a left subidealizer in $S$ at $A$, we mean that $R$ is a subring of $S, A$ is a left ideal of $S$ which is a (two-sided) ideal of $R$

Received by the editors October 7, 1986. Presented January 21, 1987, 93rd Annual Meeting of the AMS, San Antonio, Texas.

1980 Mathematics Subject Classification (1985 Revision). Primary 16A60; Secondary 16A33, $16 A 38$. 
and $A S=S$. The following theorem guarantees that such subidealizers have finite global dimension.

THEOREM 3 ([RS OR McR, THEOREM 5.13]). If $R$ is a left subidealizer in $S$ at $A$ then $\mathrm{rgl} \operatorname{dim} R \leq \mathrm{rgl} \operatorname{dim} S+\mathrm{rgl} \operatorname{dim}(R / A)+1$ and $\operatorname{lgl} \operatorname{dim} R \leq \operatorname{lgl} \operatorname{dim} S+$ $\operatorname{lgl} \operatorname{dim}(R / A)+1$.

For a particular subidealizer we have previously calculated the global dimension exactly.

THEOREM $4[\mathbf{K K}]$. Let $S$ be a ring and $B$ be an ideal of $S$. Then

$\operatorname{rgl} \operatorname{dim}\left[\begin{array}{ll}S & B \\ S & S\end{array}\right]=\max \{\operatorname{rgl} \operatorname{dim} S, \operatorname{rgl} \operatorname{dim}(S / B)+1\}$

The rings we will construct are also fiber products (pullbacks). Let $R_{1}$ be a ring with ideal $U_{1}$ and $R_{2}$ be a ring with ideal $U_{2}$ such that $R_{1} / U_{1} \simeq R_{2} / U_{2}$ under the isomorphism $\sigma: R_{1} / U_{1} \rightarrow R_{2} / U_{2}$. Let $R$ be the subring of $R_{1} \oplus R_{2}$ defined by $R=\left\{\left(r_{1}, r_{2}\right): \sigma\left(r_{1}+U_{1}\right)=r_{2}+U_{2}\right\}$. We will call such a ring $R$ the fiber product of $R_{1}$ and $R_{2}$ obtained by identifying $R_{1} / U_{1}$ with $R_{2} / U_{2}$. Fiber products which can be shown to have finite global dimension because they are also subidealizers have previously been constructed by Robson $[\mathbf{R}]$ and Stafford $[\mathbf{S t}]$.

Our examples will follow from the theorem below.

THEOREM 5. Let $T$ and $S$ be rings with $B$ an ideal of $S$ and $I$ an ideal of $T$ such that $S / B \simeq T / I$. Consider the rings

$$
R_{1}=\left[\begin{array}{cc}
T & 0 \\
T & T
\end{array}\right], \quad R_{2}=\left[\begin{array}{ll}
S & B \\
S & S
\end{array}\right]
$$

and the ideals,

$$
U_{1}=\left[\begin{array}{cc}
T & 0 \\
T & I
\end{array}\right] \unlhd R_{1}, \quad U_{2}=\left[\begin{array}{cc}
S & B \\
S & B
\end{array}\right] \unlhd R_{2} ;
$$

note that $R_{1} / U_{1} \simeq R_{2} / U_{2} \simeq S / B$. Let $R$ be the fiber product of $R_{1}$ and $R_{2}$ obtained by identifying $R_{1} / U_{1}$ with $R_{2} / U_{2}$. Then

(a) $\mathrm{rgl} \operatorname{dim} R \leq \max \{\operatorname{rgl} \operatorname{dim} T, \operatorname{rgl} \operatorname{dim} S\}+\operatorname{rgl} \operatorname{dim} T+1$.

(b) If $I$ is a nilpotent ideal of $T$, and $S$ is a prime ring with $B \neq 0$ then $\mathrm{rgl} \operatorname{dim}(R / N(R))=\max \left\{\mathrm{rgl} \operatorname{dim} R_{2}, \operatorname{rgl} \operatorname{dim}(T / N(T))\right\}$.

ProOF. Since $R$ is a left subidealizer in $\Sigma=M_{2}(T) \oplus M_{2}(S)$ at the left ideal $A=\left[\begin{array}{ll}T & 0 \\ T & 0\end{array}\right] \oplus\left[\begin{array}{ll}S & B \\ S & B\end{array}\right]$ of $\Sigma$, by Theorem 3 we have $\operatorname{rgl} \operatorname{dim} R \leq \operatorname{rgl} \operatorname{dim} \Sigma+$ $\mathrm{rgl} \operatorname{dim}(R / A)+1$, so (a) follows since $R / A \simeq T$. Furthermore, under the conditions of (b), $N(R)=\left[\begin{array}{cc}N(T) & 0 \\ T & I\end{array}\right] \oplus\left[\begin{array}{ll}0 & 0 \\ 0 & 0\end{array}\right]$ and $R / N(R) \simeq T / N(T) \oplus R_{2}$.

EXAMPLE 6 . For any preassigned integer $n \geq 3$, there exists a Noetherian affine PI ring $R$, module-finite over its center, with $K \operatorname{dim} R=1$ and $g l \operatorname{dim} R \leq 3$, but $\operatorname{gl} \operatorname{dim}(R / N(R))=n$.

PROOF. Let $S$ be the subring of $M_{n}(R)$,

$$
S=\left[\begin{array}{cccc}
k[x] & \cdots & & k[x] \\
(x) & k[x] & & \\
\vdots & \ddots & \ddots & \vdots \\
(x) & \cdots & (x) & k[x]
\end{array}\right]
$$


where $k[x]$ is a commutative polynomial ring over a field $k$, and let $B$ be the ideal of $S$,

$$
B=\left[\begin{array}{ccccc}
(x) & (x) & k[x] & \cdots & k[x] \\
& \ddots & \ddots & \ddots & \vdots \\
\vdots & & \ddots & \ddots & k[x] \\
& & & \ddots & (x) \\
(x) & & \cdots & & (x)
\end{array}\right]
$$

note that $S / B \simeq T / I$ where $T=T_{n}(k)$ and $I=(N(T))^{2}$. Using the rings and ideals of Theorem 5, by Example 1 and Theorem 4, gl $\operatorname{dim} R_{2}=\max \{1,(n-1)+1\}=n$, so applying Theorem $5, \operatorname{gl} \operatorname{dim} R \leq \max \{1,1\}+1+1=3$ and $\operatorname{gl} \operatorname{dim}(R / N(R))=$ $\max \{n, 0\}=n$.

EXAMPLE 7. There exists a Noetherian affine PI ring $R$, module-finite over its center, with $K \operatorname{dim} R=1, \operatorname{gl} \operatorname{dim} R \leq 5$, but $\operatorname{gl} \operatorname{dim}(R / N(R))=\infty$.

Proof. Choose $T$ and $I$ as in Example 2. Let

$$
S=\left[\begin{array}{ccc}
(k[x])^{*} & k[x] & k[x] \\
(x) & k[x] & k[x] \\
\left(x^{2}\right) & (x) & (k[x])^{*}
\end{array}\right],
$$

where $^{*}$ means entries agree $\bmod x$ (this ring is based on an example of K. Fields, who used power series instead of polynomials [F1]); $S$ is a $k[x]$-order in $M_{3}(k(x))$ so by $[\mathbf{A G}] \operatorname{gl} \operatorname{dim} S=\max \operatorname{gl} \operatorname{dim}\left(S_{m}\right)$, where the maximum is taken over all maximal ideals $m$ of $k[x]$. Using an argument as in [F1, p. 130], it is not hard to check that gl $\operatorname{dim} S=2$. Let $B$ be the ideal of $S$,

$$
B=\left[\begin{array}{ccc}
(x) & (x) & (x) \\
(x) & (x) & (x) \\
\left(x^{2}\right) & (x) & (x)
\end{array}\right]
$$

and note that $S / B \simeq T / I$. Using the rings and ideals of Theorem 5, by Example 2 and Theorem $4 \mathrm{gl} \operatorname{dim} R_{2}=\infty$; applying Theorem $5 \mathrm{gl} \operatorname{dim} R \leq \max \{2,2\}+2+1$ $=5$ and $\operatorname{gl} \operatorname{dim}(R / N(R))=\infty$.

We close by noting that both Examples 6 and 7 can be easily modified so that $N(R)$ is a prime ideal. Let $R^{\prime}$ be the fiber product of $T$ and $R_{2}$ be obtained by identifying $T / I$ with $R_{2} / U_{2}$. Then $R \simeq\left[\begin{array}{cc}T & 0 \\ T & R^{\prime}\end{array}\right]$, so $\operatorname{gl} \operatorname{dim} R^{\prime} \leq \operatorname{gl} \operatorname{dim} R$ by [F2]. The ring $R^{\prime}$ has the same properties as $R$.

\section{REFERENCES}

[AG] M. Auslander and O. Goldman, Maximal orders, Trans. Amer. Math. Soc. 97 (1960), 1-24. [ENN] S. Eilenberg, H. Nagao and T. Nakayama, On the dimension of modules and algebras. IV. Dimension of residue rings of hereditary rings, Nagoya Math. J. 10 (1956), 87-95.

[F1] K. L. Fields, Examples of orders over discrete valuation rings, Math. Z. 111 (1969), 126-130.

[F2] _ _ On the global dimension of residue rings, Pacific J. Math. 32 (1970), 345-349.

[GS] R. Gordon and L. W. Small, Piecewise domains, J. Algebra 23 (1972), 553-564.

[KK] E. Kirkman and J. Kuzmanovich, Matrix subrings having finite global dimension, J. Algebra 109 (1987), 74-92.

[McR] J. McConnell and J. C. Robson, Global dimension, preprint of Chapter 7 of Noncommutative Noetherian Rings, Wiley. 
[ReSS] R. Resco, L. W. Small and J. T. Stafford, Krull and global dimensions of semiprime Noetherian PI-rings, Trans. Amer. Math. Soc. 274 (1982), 285-295.

[R] J. C. Robson, Some constructions of rings of finite global dimension, Glasgow Math. J. 26 (1985), 1-12.

[RS] J. C. Robson and L. W. Small, Another change of rings theorem, Univ. of Leeds Preprint (1987).

[S] L. W. Small, Hereditary rings, Proc. Nat. Acad. Sci. U.S.A. 55 (1966), 25-27.

[St] J. T. Stafford, Global dimension of semiprime Noetherian rings, Univ. of Leeds Preprint (1987).

Department of MAThematics, WAKe Forest UNIVERSity, Winston-Salem, NORTH CAROLINA 27109 